\title{
Diversidad y Biomasa Arbórea en los Bosques Andinos del Santuario Nacional del Ampay, Apurímac - Perú
}

\section{Diversity and Arboreal Biomass in the Andean Forests of the Ampay National Sanctuary, Apurímac - Peru}

\author{
Isau Huamantupa-Chuquimaco ${ }^{1,3}$, Miguel Luza-Victorio ${ }^{1}$, Lucero Alfaro-Curitumay ${ }^{1}$, \\ Michel Ururi ${ }^{1}$, Wilfredo Huaman-Arque ${ }^{1}$, Miguel Pedraza ${ }^{1} \&$ Manuel Peralvo ${ }^{2}$.
}

${ }^{1}$ Herbario CUZ, Facultad de Ciencias, Universidad Nacional San Antonio Abad del Cusco (UNSAAC). Prolongación Av. de la Cultura, 733 Cusco, Perú.andeanwayna@gmail.com

2 Programa Bosques Andinos. Coonsorcio para el desarrollo sostenible de la ecorregión andina. (CONDESAN). Germán Alemán E12-123 y Carlos Arroyo del Río, Quito, Ecuador, 170504.

${ }^{3}$ Programa de Pós-Graduação em Botânica, Escola Nacional de Botânica Tropical, Instituto de Pesquisas Jardim Botânico de Rio de Janeiro (ENBT /JBRJ). Rua Pacheco Leão, 2040. RJ, Brasil.

\section{Resumen}

En los bosques andinos del Santuario Nacional del Ampay en el sur peruano, se evaluó la diversidad y biomasa arbórea de cuatro parcelas permanentes de $60 \mathrm{~m}$ x $60 \mathrm{~m}$. Se registraron 1920 individuos de plantas leñosas con DAP $\geq 5 \mathrm{~cm}$, pertenecientes a 35 especies, 27 géneros y 20 familias. Las especies más abundantes fueron Podocarpus glomeratus, Hesperomeles gayana y Verbesina sp. 2, que concentraron más del $56 \%$ del total. Las parcelas PBA-3 y PBA-4 fueron las más diversas con 20 especies. La estructura del bosque muestra que árboles con tallos delgados ( 5 a 15 de DAP), son dominantes. La biomasa aérea total fue de 135.40/141.49 (t ha-), de estas la parcela PBA-3 registró mayor biomasa con 52.14/52.54 (t ha-), $P$. glomeratus tuvo mayor aporte de biomasa con 100.28/104.90 (t ha-), seguidas de Vallea stipularis con $8.35 / 8.97$ (tha-') y Hesperomeles gayana con 3.40/3.78 (t ha-'). Son notablemente de baja diversidad y riqueza comparadas a otros lugares con similares altitudes como los bosques montanos de las vertientes orientales de los andes y los patrones de diversidad y biomasa aérea se asemejan a otros bosques andinos principalmente a las cuencas de ecosistemas secos del Urubamba y Paucartambo. Pero los bosques del SNA son particulares por su composición y estructura caracterizados por la dominancia de la "intimpa" Podocarpus glomeratus que a pesar de estar protegidos necesitan urgentemente de más esfuerzos de conservación por parte de la población local y autoridades del SNA que conlleven a reducir los impactos negativos vinculados a las actividades antrópicas.

Palabras clave: Diversidad, bosque andino, biomasa arbórea, intimpa, Podocarpus glomeratus.

\begin{abstract}
In the Andean forests of the National Sanctuary of Ampay in southern Peru, the diversity and arboreal aerial biomass of four permanent plots of $60 \mathrm{~m}$ x $60 \mathrm{~m}$ were evaluated. We recorded 1920 individuals of woody plants with $\mathrm{DBH} \geq 5 \mathrm{~cm}$, belonging to 35 species, 27 genera and 20 families. The most abundant species were Podocarpus glomeratus, Hesperomeles gayana and Verbesina sp. 2, which accounted for more than $56 \%$ of the total. Plots PBA-3 and PBA-4 were the most diverse with 20 species. The forest structure shows that trees with thin stems ( 5 to 15 DAP) are dominant. The total aerial biomass was of $135.40 / 141.49\left(\mathrm{t} \mathrm{ha}^{-1}\right.$ ), of these the plot PBA-3 registered greater biomass with 52.14 / $52.54\left(\mathrm{t} \mathrm{ha}^{-1}\right), P$. glomeratus had greater-
\end{abstract}


-contribution of biomass with 100.28/104.90 (t ha-1), followed by Vallea stipularis with $8.35 / 8.97(\mathrm{t}$ $\left.\mathrm{ha}^{-1}\right)$ and Hesperomeles gayana with $3.40 / 3.78\left(\mathrm{t} \mathrm{ha}^{-1}\right)$. They are remarkably low in diversity and richness compared to other places with similar altitudes as the montane forests of the eastern slopes of the Andes and the patterns of diversity and aerial biomass resemble other Andean forests mainly to the dry ecosystem basins of the Urubamba and Paucartambo. But the SNA forests are particular for their composition and structure characterized by the dominance of the "intimpa" Podocarpus glomeratus that despite being protected urgently need more conservation efforts by the local population and authorities of the NSA that lead to reduce the negative impacts linked to anthropic activities.

Key words: Diversity, Andean forest, arboreal biomass, intimpa, Podocarpus glomeratus.

\section{Introducción}

Los bosques andinos neotropicales son considerados como ecosistemas de alta riqueza biológica, que integra altos grados de endemismo en el planeta (Myers et al. 2000), y son fuentes primarias de servicios ecosistémicos vitales, razones por la que son catalogados como prioritarios para su conservación (Myers et al. 2000; Bush et al. 2007; Pennington et al. 2010). Estos bosques han sido parte importante en el sustento y albergue desde inicios de la ocupación humana en los andes (Young 2009).

Los bosques montanos del Perú abarcan cerca del 68.7 millones de hectáreas, sin embargo para el año 2010 disminuyó en 1452. 955 que equivale al 9.5\% del área deforestada, la tasa de deforestación de cerca de las 150 mil hectáreas anuales la convierte en una de las más altas en Sudamérica (Tejedor et al. 2012), siendo las mayores amenazas las actividades de ganadería y agricultura. Estos bosques montanos en el Perú están clasificados como los bosques montanos del noroeste de los andes y lasYungas del sur peruano, categorizados como amenazados dado que quedan alrededor del $50 \%$ de bosque poco impactado respecto a su estado original (Young et al. 2002, Tejedor et al. 2012).

A pesar de estas cifras alarmantes, no ha- -existido una atención acorde en el avance de la investigación técnica, ya que han sido publicados pocos trabajos de ellos con un total de 510 documentos que han sido principalmente orientados a temas taxonómicos y de sistemática vegetal (26\%), composición florística (15\%) y biogeografía (11\%), temas ecológicos como interacciones entre organismos y ambiente, su relación con el bosque y recursos con poblaciones humanas, son escasas (La torre 2016).

Los bosques montanos andinos más estudiados están inmersos en la región del Cusco con más del 50\% del total de investigaciones publicadas. En general en los bosques andinos de Perú estudios sobre la dinámica con enfoques en la biomasa, reservorios de carbono y sus relaciones con factores ambientales son escasos (Young \& León 2007; Spracklen \& Righelato 2014). En el centro y sur peruano, los datos de los inventarios forestales en parcelas permanentes son más frecuentes en la Amazonía, sin embargo, en el ámbito de los bosques montanos-andinos son pocos y los existentes han dado a conocer la presencia de altos grado de endemismo y excepcional riqueza (Reynel \& Anton 2004), los cuales incluyen además otros grupos de plantas como las epífitas y herbáceas.

En los bosques andinos del sur peruano- 
han sido estudiadas con mayor énfasis los bosques de la parte alta del Parque Nacional del Manú en el departamento del Cusco y el Santuario Histótico de Machupicchu. Sin embargo, en los bosques andinos en el departamento de Apurímac del Santuario Nacional del Ampay (SNA), son escasos, del área total del SNA, el 19\% lo conforma los rodales de Podocarpus glomeratus D. Don (Podocarpaceae) “intimpas" actualmente en peligro (EN) (PMSNA 2015). Estos bosques son considerados los mejor conservados dentro de áreas naturales protegidas del Perú, donde destaca la presencia de especies endémicas como Lupinus dicercophorus S. S m i th., Nasa $\quad 1$ i mata (J.F. Macbr.) Weigend, Nasa vargasii (J.F. Macbr.) Weigend, Pleurothallis vargasii C. Schweinf., y Monnina vargasii Ferreyra (INRENA 2006), además, de especies arbóreas como la "unka" Myrcianthes oreophila (Diels) McVaugh, "ch'uyllur" Vallea stipularis L. f., "chachacomo" Escallonia resinosa (Ruiz \& Pav.) Pers., "t'asta" Escallonia myrtilloides L. f., "huamanq'ero" Styloceras laurifolium (Willd.) Kunth, "wankartipa" Randia boliviana Rusby y “capuli-pishay” Prunus rigida Koehne (INRENA 2006), los que al igual que los "intimpales" vienen siendo diezmados drásticamente en los alrededores del SNA (PM-SNA 2015).

Por ende, es importante la implementación de estudios que den a conocer la diversidad, estructura, biomasa y las funciones que estos tienen en el ecosistema, contribuyendo de esta manera en la toma de decisiones para su protección y conservación. En este contexto el presente estudio se realizó con el objetivo principal de establecer una línea base de carbono y biodiversidad en los bosques del Santuario Nacional Ampay (PM-SNA 2003), caracterizando indicadores de diversidad, estructura, composición y la biomasa arbórea.

\section{Materiales y Métodos}

\section{Área de estudio}

El área de estudio está ubicada dentro del Santuario Nacional del Ampay (SNA), en el ámbito geopolítico del distrito de Tamburco, provincia de Abancay, departamento de Apurímac. El SNA se ubica entre las coordenadas $13^{\circ} 33^{\prime} \mathrm{LS}$ y $72^{\circ} 51^{\prime} \mathrm{LO}$, cubriendo una gradiente altitudinal de 2,900 a 5,235 msnm (Figura 1). Presenta una biotemperatura media anual máxima de $10.8^{\circ} \mathrm{C}$, y un promedio anual de precipitación de $1356 \mathrm{~mm}$ (IDMA 1998). El SNA comprende alrededor de 3853,00 hectáreas, de los cuales el 19\% lo conforma los rodales de Podocarpus glomeratus "intimpas" que se encuentra dentro de especies en peligro (INRENA 2006), estos "intimpales" actualmente son considerados como los mejores conservados en el Perú.

Las cuatro parcelas permanentes se encuentran dentro de los bosques de intimpa pertenecientes al sistema ecológico del bosque altimontano pluviestacional de las Yungas del sur peruano, caracterizado por la presencia de bosques húmedos andinos, ubicados desde los 3200 hasta los $3800 \mathrm{~m}$ (Josse et al. 2009).

\section{Metodología}

\section{Establecimiento de las parcelas permanentes}

El establecimiento de las cuatro parcelas permanentes se hizo en áreas con poco impacto antrópico y bosques con estructura clímax (tabla 1), siguiendo los protocolos establecidos por el programa de bosques andinos (Osinaga et al. 2014), que consistió en la delimitación de un área de $60 \times 60 \mathrm{~m}\left(3600 \mathrm{~m}^{2}\right)$, esta se dividió en sub-plots de 20 × 20 m (9 en cada una), se colocaron tubos pvc $(1.5 \mathrm{~m})$ en cada intersección, en cada parcela se midió, plaqueó 


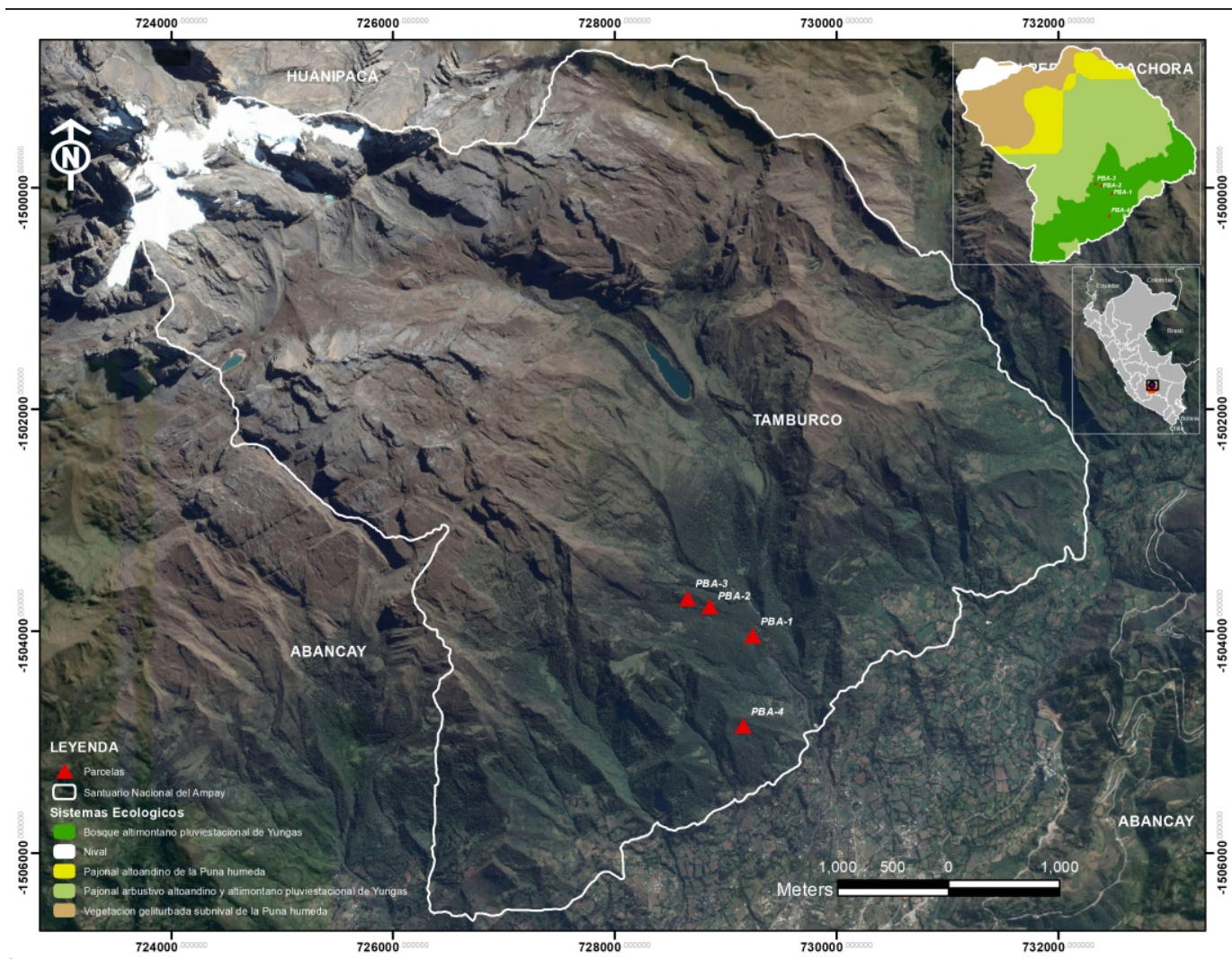

Figura 1. Ubicación de las cuatro parcelas en el Santuario Nacional del Ampay, en la provincia de Abancay, departamento de Apurímac-Perú.

-y marcó a cada individuo con Dap $\geq 5 \mathrm{~cm}$, a $1.3 \mathrm{~m}$ de altura del suelo como punto óptimo de medida (POM). La evaluación incluyó a lianas, hemiepífitas y árboles. Las medidas de los diámetros y alturas se basaron en los protocolos establecidos por Phillips \& Baker (2009).

Se realizaron colectas de los especímenes evaluados, la posterior identificación y deposito se hizo en el herbario CUZ de la Universidad Nacional de San Antonio Abad del Cusco.

\section{Análisis de datos}

El tratamiento taxonómico de las familias sigue los planteamientos filogenéticos del-
Tabla 1. Datos de ubicación de parcelas permanentes.

\begin{tabular}{lccc}
\hline Parcela & $\begin{array}{c}\text { Altitud } \\
\text { (msnm) }\end{array}$ & $\begin{array}{c}\text { Coordenadas } \\
\text { UTM }\end{array}$ & $\begin{array}{c}\text { Pendiente } \\
(\mathbf{\%})\end{array}$ \\
\hline PBA-1 & 3296 & 18 L 729244 8495958 & 50 \\
PBA-2 & 3340 & 18 L 728857 8496223 & 50 \\
PBA-3 & 3440 & 18 L 728652 8496296 & 60 \\
PBA-4 & 3260 & 18L 7291578495146 & 60 \\
\hline
\end{tabular}

-Grupo de Filogenia de las Angiospermas (APG IV 2016) y se usó la bibliografía correspondiente. Además se consultó en línea las siguientes páginas:

http: / / w w w.tropicos.org, http / / ww w. the plantlist.org y http:/ / www.fieldmuseum.org. 


\section{Diversidad}

Para cada parcela los valores de diversidad fueron calculados de dos maneras. Primero se calcularon los valores reales de riqueza contando el número de especies que fueron encontrados en cada parcela y calculándose el índice de diversidad de Alfa de Fisher por parcela, la cual ha demostrado ser consistente a variaciones de abundancia comparado con otros índices de diversidad y es cada vez más usado en comparaciones de diversidad florística entre parcelas (Condit et al. 1998; Phillips \& Miller 2002; Ter Steege et al. 2003). Para estos cálculos se utilizó el software estadístico Past vers. 3.14 (Hammer et al. 2001).

\section{Estructura y composición}

Para cada parcela se calculó la abundancia relativa, dominancia relativa, frecuencia relativa y sumando estas el índice de Valor de Importancia por especie (IVI) (Curtis \& McIntosh 1951) usando las siguientes formulas: Abundancia Relativa: DeRj = $100 \times$ Dej/ $\sum$ Dej; Dominancia Relativa: DoRj $=100 \times$ Doj $/ \sum$ Doj; Frecuencia Relativa: FRj $=100 \times \mathrm{Fj} / \sum \mathrm{Fj} ; \mathrm{y}$ el Índice de Valor de Importancia: $\mathrm{IVIj}=\mathrm{DeRj}+\mathrm{DoRj}+\mathrm{FRj}$, Dónde: Dej es el número total de tallos de la especie j en todas las parcelas, Doj es el área basal total de la especie $\mathrm{j}$ en todas parcelas, y Fj es el número de parcelas donde esta presenta la especie j. Para observar las variaciones de los diámetros de los árboles según las clases diamétricas y de alturas para los géneros e individuos en cada parcela se construyeron los diagramas de cajas, en los casos de variaciones amplias como del DAP se transformaron a Logaritmo. Estos análisis fueron realizados con el software estadístico $\mathrm{R}$ v3.2.1 (Equipo de desarrollo de R, 2013) y Past vers. 3.14 (Hammer et al. 2001).

\section{Diversidad Beta}

El porcentaje de similitud florística entre parcelas fue determinado calculando los coeficientes de similitud de Bray-Curtis, usando datos de abundancia (Magurran 1988). Para el análisis de agrupamiento se usó el índice de distancia de Bray-Curtis (Sorensen cuantitativo) con datos de abundancia para calcular la similitud florística entre parcelas y el promedio de grupos (UPGMA) como método de enlace entre grupos y pares de grupos. Los análisis de similitud florística fueron realizados con el software estadístico Past vers. 3.14 (Hammer et al. 2001).

\section{Estimación de la Biomasa Aérea}

La biomasa aérea (AGB-estimada) se estimó en base a dos ecuaciones alométricas propuestas por Chave et al. (2005) y Chave et al. (2014), que consideran para cada árbol, el diámetro (D) y la altura evaluada en campo $(\mathrm{H})$, y la densidad ( $p$ ) de la madera. Estos dos modelos alométricos son aplicados para árboles tropicales en bosques montanos y para bosques que reciben precipitación entre 1500 a $3200 \mathrm{~mm}$ anuales (tabla 2). La densidad ( $p$ ) para cada árbol fue estimada utilizando los datos reportados para los bosques neotropicales en el Global Wood Density Database, para las especies o géneros que no se hallaron sus densidades se usó una media global a nivel de género o familia $\left(0.62 \mathrm{~g} / \mathrm{cm}^{3}\right)$ (Chave et al. 2009 y Zanne et al. 2009).

Para observar las variaciones de las muestras de biomasa en cada parcela se construyeron diagramas de cajas y para conocer si existen diferencias en las biomasas entre las cuatro parcelas y los géneros, en el caso de variaciones amplias de valores de biomasa se transformaron a Logaritmo. 
Tabla 2. Ecuaciones alométricas utilizadas para la estimación de la Biomasa aérea.

\begin{tabular}{|c|c|c|}
\hline Autor & Ecuación & Fundamento \\
\hline Chave et al. (2005). & $\begin{array}{l}A G B=\text { ex p }-2.977+\ln p i D i 2 H i=0.0509 * \\
\text { piDi2 Hi }\end{array}$ & $\begin{array}{l}\text { Bosques estacionales }(1500-3500 \mathrm{mma}-1 \\
\text { Moist (Height) }\end{array}$ \\
\hline Chave et al. (2014). & $A G B=0.0673 *($ piDi2Hi $) 0.976$ & Moist (Height) \\
\hline
\end{tabular}

También se realizó el análisis comparativo no paramétrico de Kruskall-Wallis y el análisis post-hot de Bonferroni que muestra las diferencias entre cada una de las muestras de Biomasa. Para todos los análisis estadísticos se usaron el software estadístico R v3.2.1 (Equipo de desarrollo de R, 2013) y Past vers. 3.14 (Hammer et al. 2001).

\section{Resultados}

\section{Diversidad y composición}

Se evaluaron 1920 individuos de plantas leñosas con Dap $\geq 5 \mathrm{~cm}$, que pertenecen a 35 especies, 20 familias y 27 géneros. Fueron identificados el 70\% de las especies. Las familias más diversas fueron Asteraceae con seis especies, Solanaceae y Verbenaceae con cuatro cada una, y Araliaceae con tres. Los géneros más ricos en especies fueron Oreopanax $y$ Solanum con tres, seguida de Cytharexylum, Myrsine, Duranta, y Verbesina con dos cada una, el resto de géneros estuvieron representadas por una sola especie. Las parcelas más diversas fueron PBA-3 y PBA-4, con 20 especies. El índice de Alfa de Fisher fue de 4.37 (PBA-3) y 4.1 (PBA-4) respectivamente, seguida por las parcelas PBA-2 con 18 especies y 3.7 de índice deAlfa de Fisher. (Tabla 3).

Las tres especies más abundantes fueron: Podocarpus glomeratus con 876 individuos, Hesperomeles gayana (102) y Verbesina sp. 2 con 100. Estas tres especies concentran más del 56 $\%$ (1078) del total de tallos evaluados en las 4 parcelas (tabla 4$)$.
Tabla 3. Datos de estructura y diversidad de las cuatro parcelas permanentes en el SNA.

\begin{tabular}{lcccc}
\hline Parcela & N\# Familias & N\# Especie & $\begin{array}{c}\text { N\# } \\
\text { Individuos }\end{array}$ & $\begin{array}{c}\text { Diversidad Alfa } \\
\text { Fisher }\end{array}$ \\
\hline PBA-1 & 9 & 14 & 272 & 3.12 \\
PBA-2 & 12 & 18 & 476 & 3.7 \\
PBA-3 & 15 & 20 & 420 & 4.37 \\
PBA-4 & 14 & 20 & 752 & 4.1 \\
\hline
\end{tabular}

La clase diamétrica que más individuos incluyó fue la de 5 a $15 \mathrm{~cm}$ de diámetro a la altura de pecho (DAP), seguidas de las clases 15.1 a $25 \mathrm{~cm}$ de DAP (Figura 4). En la parcela PBA-3 se registró un individuo de $393.74 \mathrm{~cm}$ de DAP, el cual contribuyó el 60.3\% (3.9 m²) del total de área basal en la parcela.

En las clases de alturas la categoría de 6 a 10 $\mathrm{m}$, incluyó 918 individuos (48\% del total), pocos individuos se observaron por encima de los 15 metros (figura 3).

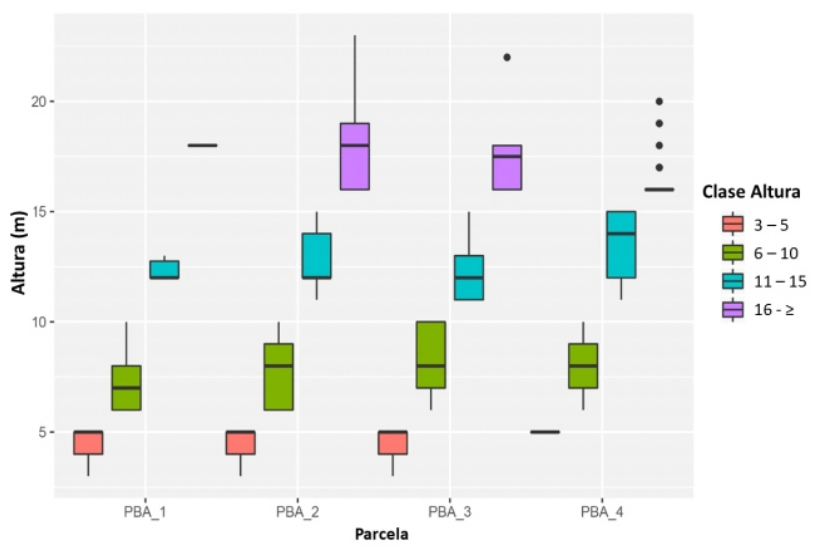

Figura 3. Distribución de los individuos según las clases de alturas en las cuatro parcelas. 
Tabla 4. Listado de especies y sus abundancias de las cuatro parcelas evaluadas en el SNA.

\begin{tabular}{|c|c|c|c|c|c|c|}
\hline Familia & Especie & PBA-1 & PBA-2 & PBA-3 & PBA-4 & $\mathrm{N}^{\circ}$ Colecta \\
\hline Aquifoliaceae & Ilex sp. 1 & & 9 & & & IHC 19410A \\
\hline \multirow[t]{3}{*}{ Araliaceae } & Oreopanax aff. cuspidatus Harms & & 1 & 8 & 2 & IHC 19410B \\
\hline & $\begin{array}{l}\text { Oreopanax capitatus (Jacq.) Decne. \& } \\
\text { Planch. }\end{array}$ & & & & 23 & LA 14 , LA 17 \\
\hline & Oreopanax cuspidatus Harms & & 1 & & 1 & LA 13 \\
\hline \multirow[t]{6}{*}{ Asteraceae } & Baccharis buxifolia (Lam.) pers. & 22 & & 8 & 8 & IHC 19409, LA 04 \\
\hline & Barnadesia Horrida Muschl. & & & 1 & & Obs. \\
\hline & Gynoxys sp. 1 & & & 28 & 2 & IHC 19408B \\
\hline & Mikania sp. 1 & & 1 & & & Obs. \\
\hline & Verbesina sp. 1 & 2 & 40 & 9 & 2 & IHC 19404A \\
\hline & Verbesina sp. 2 & & 100 & & & IHC 19404B \\
\hline Berberidaceae & Berberis sp. 1 & & & 15 & 4 & IHC 19408A \\
\hline Buxaceae & Styloceras laurifolium (Willd.) Kunth & & 1 & & & Obs. \\
\hline Campanulaceae & Siphocampylus veteranus E. Wimm. & & & 1 & 1 & IHC 19398B \\
\hline Clusiaceae & Clusia aff. weberbaueri Engl. & & & & 11 & LA 10 \\
\hline Cornaceae & Cornus peruviana J.F. Macbr. & & & & 12 & LA 16 \\
\hline Elaeocarpaceae & Vallea stipularis $\mathrm{L} . \mathrm{f}$. & 5 & 6 & 56 & 23 & IHC $19398 \mathrm{~A}$ \\
\hline Escalloniaceae & Escallonia resinosa (Ruiz \& Pav.) Pers. & & & 1 & & Obs. \\
\hline Lamiaceae & Aegiphila mortonii Moldenke & & 53 & 2 & & Obs. \\
\hline Melastomataceae & Miconia sp. 1 & & & 1 & & IHC 19402 \\
\hline Myrtaceae & Myrcianthes oreophila (Diels) McVaugh & 11 & 9 & 13 & 44 & IHC 19414, LA 06 \\
\hline Podocarpaceae & Podocarpus glomeratus D. Don & 161 & 106 & 212 & 397 & IHC 19412 \\
\hline Polygonaceae & Muehlenbeckia sp. 1 & 1 & & & & IHC 19405 \\
\hline \multirow[t]{2}{*}{ Primulaceae } & Myrsine andina (Mez) Pipoly & & 5 & 29 & 25 & LA 15 \\
\hline & Myrsine aff. pseudocrenata (Mez) Pipoly & & & & 1 & LA 26 \\
\hline \multirow[t]{2}{*}{ Rosaceae } & $\begin{array}{l}\text { Hesperomeles gayana (Decne.) J.F. } \\
\text { Macbr. }\end{array}$ & 3 & & 7 & 92 & IHC 19411 \\
\hline & Prunus brittoniana Rusby & 2 & & 7 & 21 & IHC 19407 \\
\hline Rubiaceae & Randia boliviana Rusby & 24 & 5 & 2 & 12 & IHC 19397 \\
\hline \multirow[t]{4}{*}{ Solanaceae } & Saracha punctata Ruiz \& Pav. & & 4 & & & IHC 19406 \\
\hline & Solanum maturecalvans Bitter & 10 & & & & IHC 19400 \\
\hline & Solanum sp. 1 & & 44 & 1 & & IHC 19413 \\
\hline & Solanum tomentosum Philippi f. & 1 & & & & IHC 19399 \\
\hline \multirow[t]{4}{*}{ Verbenaceae } & Citharexylum herrerae Mansf & 19 & 8 & 4 & & IHC 19403 \\
\hline & Citharexylum laurifolium Hayek & & & & 2 & LA 03 \\
\hline & Duranta armata Moldenke & 1 & 64 & 15 & & IHC 19401A \\
\hline & Duranta mandonii Moldenke & 10 & 19 & & 41 & IHC 19401B, LA 01 \\
\hline Total & & 272 & 476 & 420 & 752 & \\
\hline
\end{tabular}



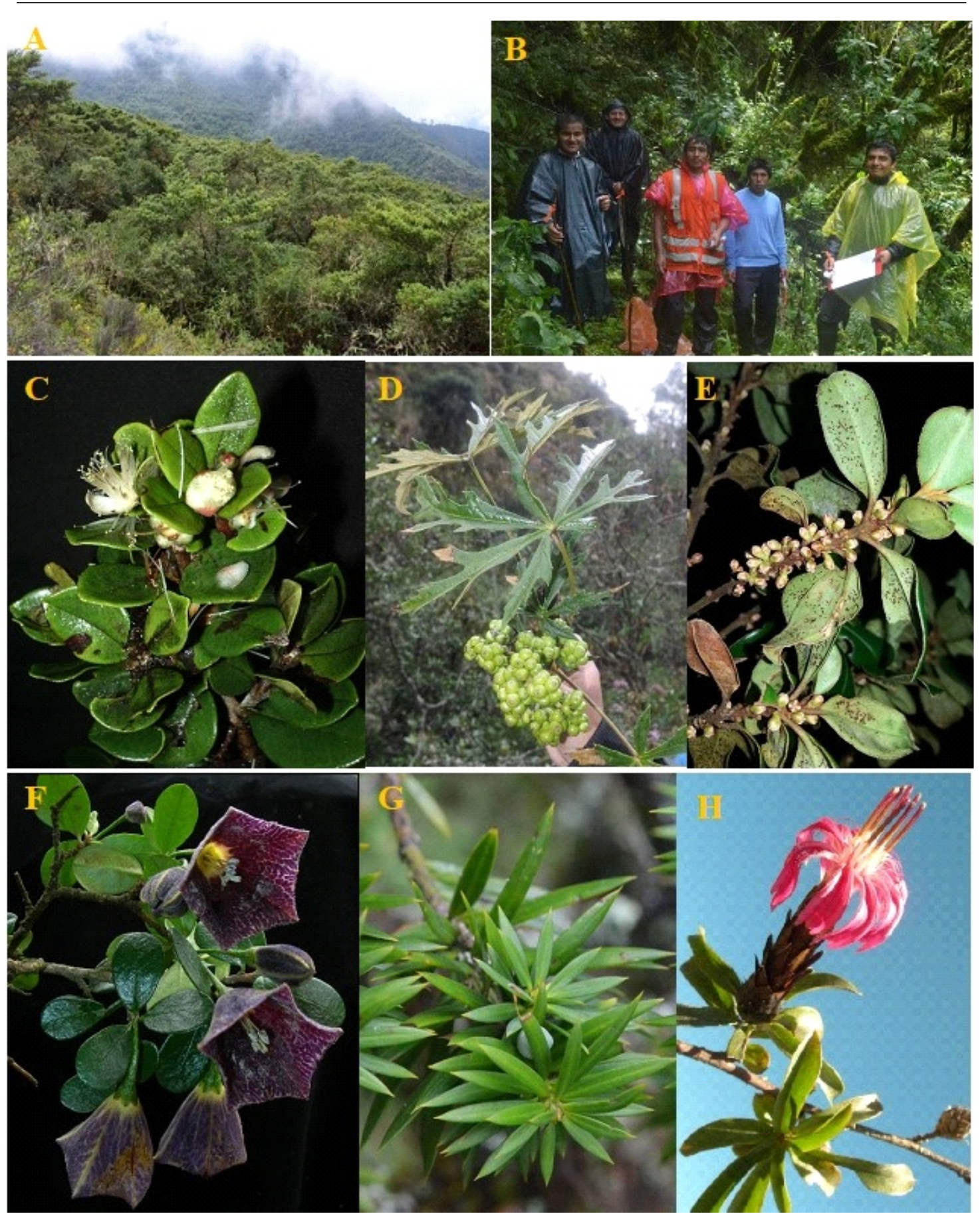

Figura 2. A. Zonas de instalación de las parcelas en el Santuario Nacional del Ampay, B. Equipo de trabajo, C. Myrcianthes oreophylla, D. Oreopanax cuspidatus, E. Myrsine andina, F. Saracha punctata, G. Podocarpus glomeratus y H. Barnadesia horrida. 
En las 4 parcelas a nivel de géneros se observó (Figura 4), que las de mayores portes fueron Podocarpus con la especie Podocarpus glomeratus, Oreopanax (Oreopanax cuspidatus y Vebesina con (Verbesina sp1).
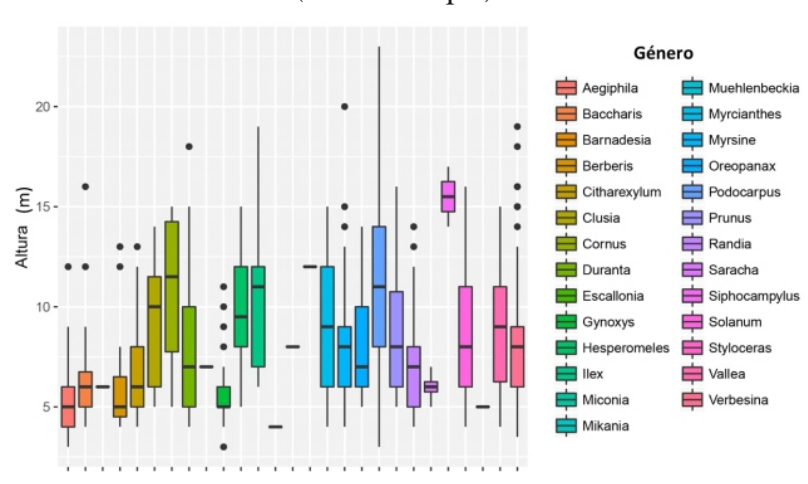

Figura 4. Distribución de los géneros según las variaciones de alturas en las cuatro parcelas.

A nivel de DAP, las mayores variaciones se observaron en las clase diamétricas de 45 a más, donde P. glomeratus fue la representativa (Figura 5).

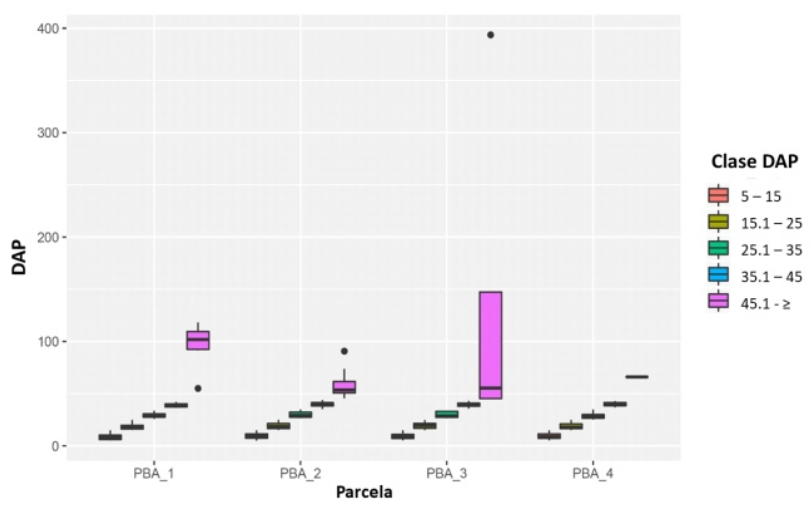

Figura 5. Distribución de los individuos según las clases diamétricas en cada parcela.

En las 4 parcelas a nivel de géneros se observó (Figura 6), que los diámetros mayores lo obtuvieron Podocarpus con la especie Podocarpus glomeratus, Vebesina con (Verbesina sp1) y Gynoxys con Gynoxys sp. 1.

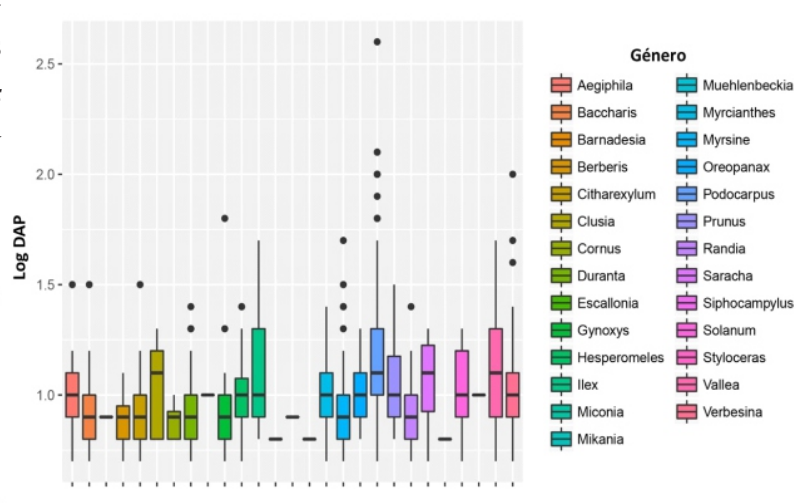

Figura 6. Distribución de los géneros mostrando las variaciones de los DAP en las cuatro parcelas (DAP transformado a Log.).

En la PBA-1 se evaluaron 272 individuos, que incluyeron nueve familias con 14 especies, de estas Podocarpus glomeratus fue la más abundante con 161 individuos, seguidas de Randia boliviana con 24 y Baccharis buxifolia con 22 individuos. P. glomeratus contribuyó con el $75.839 \%$ (31.21 $\left.\mathrm{m}^{2}\right)$ del área basal total, y fue la de mayor índice de valor de importancia con 249.58. En la parcela PBA-2 se evaluaron 476 individuos, que incluyeron 12 familias con 18 especies, de estas Podocarpus glomeratus fue la más abundante con 106 individuos, seguidas de Verbesina sp. 2 con 100 y Duranta armata con 64 individuos. P. glomeratus contribuyo con el $52.1 \%$ del área basal total, y fue la de mayor índice de valor de importancia con 174.4. En la parcela PBA-3 se registraron 420 individuos, que incluyeron 14 familias con 20 especies, de estas Podocarpus glomeratus fue la más abundante con 212 individuos, seguidas de Vallea stipularis con 56 y Myrsine andina con 29 individuos. P. glomeratus contribuyó con el 50.48\% del área basal total, y fue la de mayor índice de valor de importancia con 232.86 y en la parcela PBA-4 se registraron 752 individuos, que incluyeron 14 familias con 20 especies, de estas- 
Tabla 5. Valores de índice de importancia para cada parcela evaluada en el SNA.

\begin{tabular}{|c|c|c|c|c|c|}
\hline Familia & Especie & PBA-1 & PBA-2 & PBA-3 & PBA-4 \\
\hline Aquifoliaceae & Ilex sp. 1 & 0 & 12.61 & 0 & 0 \\
\hline \multirow[t]{3}{*}{ Araliaceae } & Oreopanax capitatus & 0 & 0 & 0 & 11.80 \\
\hline & Oreopanax cuspidatus & 0 & 3.82 & 8.49 & 2.26 \\
\hline & Oreopanax sp. 1 & 0 & 0 & 0 & 1.20 \\
\hline \multirow[t]{6}{*}{ Asteraceae } & Baccharis buxifolia & 18.08 & 0 & 7.17 & 7.14 \\
\hline & Barnadesia horrida & 0 & 0 & 1.48 & 0 \\
\hline & Gynoxys sp. 1 & 0 & 0 & 17.67 & 2.28 \\
\hline & Mikania sp. 1 & 0 & 1.80 & 0 & 0 \\
\hline & Verbesina sp. 1 & 4.92 & 31.95 & 7.44 & 2.55 \\
\hline & Verbesina sp. 2 & 0 & 38.33 & 0 & 0 \\
\hline Berberidaceae & Berberis sp. 1 & 0 & 0 & 8.82 & 4.51 \\
\hline Buxaceae & Styloceras laurifolium & 0 & 1.83 & 0 & 0 \\
\hline Campanulaceae & Siphocampylus veteranus & 0 & 0 & 1.47 & 1.12 \\
\hline Clusiaceae & Clusia af. weberbaueri & 0 & 0 & 0 & 8.42 \\
\hline Cornaceae & Cornus peruviana & 0 & 0 & 0 & 5.97 \\
\hline Elaeocarpaceae & Vallea stipularis & 11.52 & 8.72 & 33.18 & 12.11 \\
\hline Escalloniaceae & Escallonia resinosa & 0 & 0 & 1.49 & 0 \\
\hline Lamiaceae & Aegiphila mortonii & 0 & 20.25 & 2.94 & 0 \\
\hline Melastomataceae & Miconia sp. 1 & 0 & 0 & 1.48 & 0 \\
\hline Myrtaceae & Myrcianthes oreophila & 17.25 & 8.89 & 11.28 & 17.25 \\
\hline Podocarpaceae & Podocarpus glomeratus & 167.95 & 88.29 & 143.84 & 134.42 \\
\hline Polygonaceae & Muehlenbeckia sp. 1 & 2.44 & 0 & 0 & 0 \\
\hline \multirow[t]{2}{*}{ Primulaceae } & Myrsineaff. pseudocrenata & 0 & 0 & 0 & 1.19 \\
\hline & Myrsine andina & 0 & 12.09 & 17.95 & 18.32 \\
\hline \multirow[t]{2}{*}{ Rosaceae } & Hesperomeles gayana & 3.45 & 0 & 5.85 & 28.28 \\
\hline & Prunus brittoniana & 4.92 & 0 & 7.62 & 12.60 \\
\hline Rubiaceae & Randia boliviana & 23.03 & 5.85 & 3.02 & 9.94 \\
\hline \multirow[t]{4}{*}{ Solanaceae } & Saracha punctata & 0 & 2.99 & 0 & 0 \\
\hline & Solanummaturecalvans & 12.24 & 0 & 0 & 1.12 \\
\hline & Solanum sp. 1 & 0 & 19.45 & 0 & 0 \\
\hline & Solanum tomentosum & 2.51 & 0 & 0 & 0 \\
\hline \multirow[t]{4}{*}{ Verbenaceae } & Citharexylum herrerae & 18.92 & 5.37 & 5.91 & 0 \\
\hline & Citharexylum laurifolium & 0 & 0 & 0 & 2.35 \\
\hline & Duranta armata & 2.43 & 28.02 & 11.43 & 0 \\
\hline & Duranta mandonii & 10.34 & 9.75 & 0 & 15.16 \\
\hline Total & & 300.00 & 300.00 & 300.00 & 300.00 \\
\hline
\end{tabular}

Podocarpus glomeratus fue la más abundante con 397 individuos, seguidas de Hesperomeles gayana con 92 y Myrsine andina con 52 individuos. P. glomeratus contribuyo con el $52.8 \%$ del área basal total, y fue la de mayor índice de valor de importancia con 134.6.8 (tabla 5).

\section{Diversidad Beta}

El análisis de agrupamiento del UPGMA mediante el índice de similitud Bray Curtis, exhibe la formación de un grupo consistente representada por las parcelas PBA-1, PBA-3 y PBA-4 
-que con un coeficiente de similitud que osciló entre 0.61 y 0.42 , se interpreta como similitud alta. Entre las tres en conjunto comparten 8 especies: Myrcianthes oreophila, Podocarpus glomeratus, Randia boliviana, Vallea stipularis, Verbesina sp. 1, Baccharis buxifolia, Hesperomeles gayana y Prunus brittoniana. Este grupo difiere de PBA-2 con un coeficiente de 0.26 , que representa una baja similitud compartiendo pocas especies.

\section{Biomasa Aérea}

Para las cuatro parcelas se totalizó 192.40 (1.44 $\left.\mathrm{t} \mathrm{ha}^{-1}\right)$, con la ecuación alométrica de Chave et al. (2005) y 202.49 (1.44 t ha-1), con la ecuación de Chave et al. (2014). De estas la PBA-3 fue la de mayor biomasa aérea con 52.14/52.54 ( $\left.\mathrm{t} \mathrm{ha}^{-1}\right)$, seguida de la PBA-4 con 33.04.08/36.34 (t ha $\left.{ }^{-1}\right)$ (tabla 9, figura 7).

Tabla 9. Valores de los parámetros estructurales de Biomasa.

\begin{tabular}{lccc}
\hline \multicolumn{1}{c}{ Parcela } & $\begin{array}{c}\text { Área Basal } \\
\left(\mathbf{m}^{\mathbf{2}}\right)\end{array}$ & $\begin{array}{c}\text { AGB }\left(\mathrm{t} \mathrm{ha}^{-1}\right) \\
(\text { Chaveet } \text { al. 2005) }\end{array}$ & $\begin{array}{c}\text { AGB }\left(\mathrm{t} \mathrm{ha}{ }^{-1}\right) \\
(\text { Chaveet al. 2014) }\end{array}$ \\
\hline PBA-1 & 9.54 & 19.91 & 20.92 \\
PBA-2 & 11.46 & 29.92 & 32.19 \\
PBA-3 & 20.17 & 52.14 & 52.54 \\
PBA-4 & 13.72 & 33.04 & 36.34 \\
Prom. \pm D.E & $13.72 \pm 566$ & $48.27 \pm 24.65$ & $50.05 \pm 23.96$ \\
\hline Total & $\mathbf{5 4 . 8 9}$ & $\mathbf{1 3 5 . 0 0 4}$ & $\mathbf{1 4 1 . 9}$ \\
\hline
\end{tabular}

Según las dos ecuaciones alométricas para las cuatro parcelas la especie que contribuyó con mayor biomasa fue Podocarpus glomeratus con 100.28/104.90 ( $\left.\mathrm{t} \mathrm{ha}^{-1}\right)$, por tanto fue la especie que aportó con más del $73 \%$ de la biomasa total (Figura 7) y que las variaciones de sus promedios son parecidos, las otras especies de mayor contribución fueron: Vallea stipularis con 8.35/8.97 ( $\left.\mathrm{t} \mathrm{ha}^{-1}\right)$, Hesperomeles gayana con $3.40 / 3.78\left(\mathrm{t} \mathrm{ha}^{-1}\right)$ y Myrcianthes oreophylla con 2.94/3.26( $\left.\mathrm{tha}^{-1}\right)$.

En las 4 parcelas en conjunto, a nivel de géneros se halló que los de mayor variación de biomasa- -acumulada son: Podocarpus con la especie Podocarpus glomeratus, seguidas de Vallea con Vallea stipularis, Verbesina con Verbesina sp. 1 e Ilex con la especie Ilex sp. 1 (Figura 7).

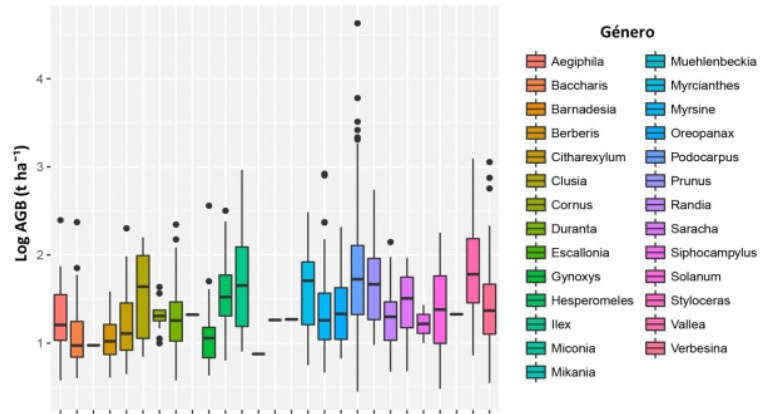

Figura 7. Distribución de los géneros mostrando la variación de biomasa, nótese que Podocarpus glomeratus fue la que concentro la mayor biomasa y variabilidad (valores de Biomasa Chave et al. 2014 en Log.).

La variación de la biomasa en las 4 parcelas estuvo concentrada principalmente en la clase diamétrica de 45 a más (figura 8, 9), de estas destaca la presencia de Podocarpus glomeratus en la PBA-3 con el valor más alto, donde un solo individuos con alto valor de DAP (mayor a $3 \mathrm{~m}$ ) hace que esta parcela tenga la mayor concentración de biomasa.

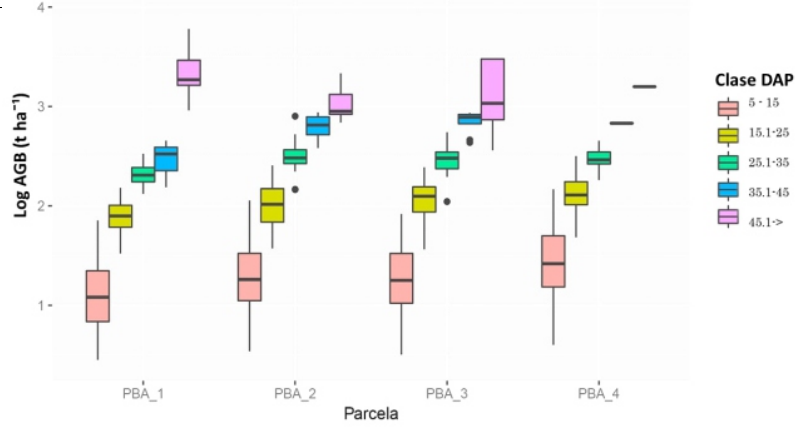

Figura 8. Diagrama de Cajas comparativas de las biomasas y sus márgenes de variación en cada parcela según las clases diamétricas con la ecuación alométrica Chave et al. (2014). 


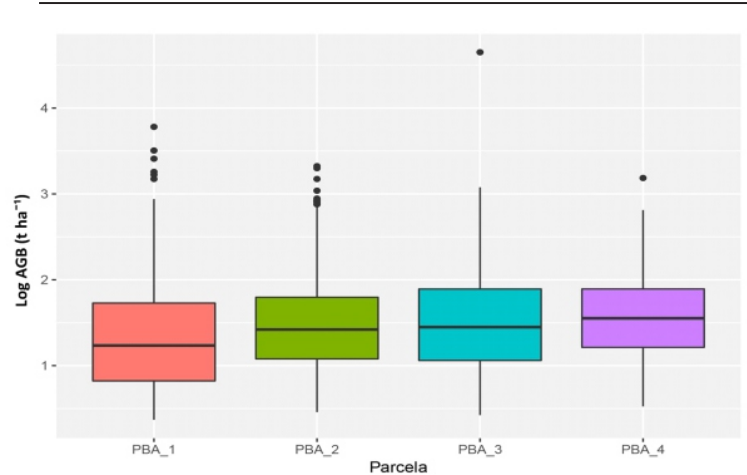

Figura 9. Diagrama de cajas comparativas de las biomasas y sus márgenes de variación en cada parcela con la Ecuación alométrica Chave et al. (2014).

La comparación de las biomasas mediante la prueba no paramétrica de Kruskal-Wallis revela que la cantidad de biomasa entre las cuatro parcelas no muestran diferencias significativas $(p=0.126)$ aplicadas para ambas ecuaciones, y en la comparación a posteriori con la prueba de Bonferroni se corrobora que no existe diferencias entre las tres poblaciones a través de las biomasas (tabla 10).

Tabla 10. Estadísticos de la prueba no paramétrica post-hot de Bonferroni. Matriz superior de valores de la ecuación alométrica de Chave et al. 2014 y matriz inferior de Chave et al. 2005.

\begin{tabular}{l|cccc}
\multicolumn{1}{l}{} & PBA-1 & PBA-2 & PBA-3 & PBA-4 \\
\hline PBA-1 & & 0.77 & 0.66 & 0.17 \\
PBA-2 & 0.07 & & 0.13 & 0.47 \\
PBA-3 & 0.66 & 0.13 & & 0.31 \\
PBA-4 & 0.17 & 0.47 & 0.32 &
\end{tabular}

\section{Discusiones}

\section{Diversidad y composición}

El presente estudio representa un avance en el conocimiento de la diversidad y dinámica arbórea del departamento de Apurímac y el sur peruano, que será una excelente línea de base para el monitoreo de la dinámica de los bosques
- nativos a ndinos. Ya que desafortunadamente en general los bosques montanos andinos son uno de los hábitats pobremente conocidos hasta ahora (Gentry 1995; Webster 1995) y más aún en los bosques peruanos.

Las 35 especies arbóreas aquí reportadas indican que se logró registrar el 70.5\% del total de especies forestales reconocidas para el SNA como refiere Hostning-Palomino (1997). La diversidad y riqueza de especies muestra que las familias Asteraceae, Solanaceae y Verbenaceae fueron las más diversas, estos patrones son similares a los reportados por los bosques de la cuenca del Urubamba (Tupayachi 2005) y el Mapacho en la provincia de Paucartambo en la región sur peruana (Observación personal), cuencas caracterizadas por presentar regímenes de temperatura y precipitación de ecosistemas secos. La diversidad y riqueza en las cuatro parcelas, muestra que algunos lugares pueden mostrar relativas diferencias como sucede entre la PBA-3 y PBA-4 que presentan 20 especies cada una y PBA1-con 14 especies -y PBA-2 con 18 especies, lo cual está relacionada directamente con el estado de conservación, una zona con claros de bosque originados aparentemente por impacto humano anterior a la creación del SNA. Esta relativa diferencia se corrobora por el índice de diversidad de Alfa de Fisher donde PBA-3 y PBA-4 presentan 4.37 y 4.1 respectivamente.

En general la vegetación en el ámbito del SNA está compuesta por arbustos de hoja caduca, hierbas anuales abundantes, árboles pequeños, arbustos espinosos que dan una fisonomía de bosque con porte bajo, el dosel principal está dominado por Podocarpus glomeratus. 
La composición de especies está representada por géneros y especies típicas de bosques montanos húmedos inter andinos como: Myrcianthes oreophylla (Myrtaceae), Oreopanax spp (Araliaceae), Gynoxys spp (Asteraceae), Vallea stipularis (Elaeocarpaceae), Escallonia resinosa (Escalloniaceae, patrón observado también en las partes altas de la cuenca del Urubamba como se constata en Tupayachi (2005), que a diferencia de las poblaciones de Podocarpus glomeratus en esta cuenca son escasas. En la cuenca del MapachoPaucartambo y los bosques de Alto Timpiñari en el distrito de Pichari (Cusco) son quizá los únicos lugares comparables donde se pueden hallar a poblaciones saludables de esta especie como sucede en el SNA. Otras formas de hábito como la presencia escasa de lianas son corroboradas para varios estudios similares en bosques altoandinos húmedos donde aparentemente debido a las condiciones de bajas temperaturas, y alta humedad que desfavorecen a este tipo de hábitos (Gentry 1995, Tupayachi 2005).

$\mathrm{Al}$ agrupar nuestros datos de las 4 parcelas evaluadas que representa a un poco más de 1 ha, se puede notar que los bosques del SNA presentan una diversidad arbórea relativamente baja, que por ejemplo comparado a la parte alta del Parque Nacional del Manu en la localidad de Wayquecha, Rivera (2007) registra a $2900 \mathrm{msnm}$ la riqueza de 68 especies y 20 familias en 1 ha, donde las familias más diversas fueron Sabiaceae con 9 especies, Lauraceae (7) y Cyatheaceae (7), donde las especies más representativas con mayor IVI fueron Weinmannia latifolia, Clusia of poepiggiana, Prunus integrifolia, Weinmannia crassifolia, Myrsine coriacea y Miconia lívida, de las cuales ninguna familia y especies se han registrado en el SNA.
Sin embargo, otra área en la zona alta del PNM en el sector trocha Unión a 3450 msnm Nina (2014) registra cifras de riqueza más semejantes al SNA, quién en una parcela de 1 ha ubicada, refiere a 593 individuos con 43 especies y 20 familias y con Weinmannia cochensis, Miconia cf. denticulata y de Hedyosmum scabrum como las especies de mayor IVI pero tampoco alguna de ellas están en común con el SNA. En otros bosques a altitudes similares como la parcela de 1 ha en Río Abiseo a 3350 msnm las cifras de riqueza y diversidad se asemejan relativamente a nuestra área, donde se registró la presencia de 1801 individuos 45 especies y 25 familias (Young 1998b). En el centro peruano en la provincia de Oxapampa Pasco en la localidad de Abra Yanachaga, Perea (2005), en una parcela de 1 ha, registra 43 especies en 19 familias con dap $\geq 10 \mathrm{~cm}$, donde las especies de mayor IVI fueron Weinmannia microphylla, Clusia multiflora y Hedyosmum cuatrecazanum, que tampoco estuvieron presentes en nuestroárea de estudio.

En los bosques andinos de Ecuador en la provincia de Chimborazo Caranqui (2014), en parcelas de $0.1 \mathrm{ha}$, con DAP $\geq 5 \mathrm{~cm}$, en 7 transectos distribuidas en gradientes de 2780 a 3600 registro 71 especies de árboles con promedios de 21 especies por lugar., de estas refiere a Oreopanax ecuadorensis, Vallea stipularis, Miconia bracteolata y Grosvenoria campii como las especies de mayor IVI. Comparando al SNA en común esta Vallea stipularis en tanto las otras dos están representadas por sus equivalentes Oreopanax cuspidatus y Miconia sp. 1., sin embargo, en una de las localidades ubicada en la vertiente occidental andina (Colta) Podocarpus glomeratus fue la de mayor IVI, mostrando que esta especie puede encontrarse como dominante en otras latitudes de los bosques andinos. Otras especies con menores valores de 
de IVI en común con el SNA fueron Myrsine andina, Styloceras laurifolium y otros géneros equivalentes, entre ellas Clusia, Escallonia, Hesperomeles, Gynoxys, Solanum, Prunus, Berberis y Myrcianthes.

Así mismo Gentry (1995), hace referencia que, en los bosques montanos altoandinos en el sur y norte peruano por encima de los 3000 msnm, las familias Lauraceae, Melastomataceae y Primulaceae son las más diversas, lo cual en general al comparar con nuestros resultados difieren notablemente dado que las familias Lauraceae y Cunnoniaceae en el SNA no fueron registrados. Esta diferencia es notable por la ubicación del SNA asentada en la vertiente occidental de los andes donde factores como la presencia de temperatura y precipitación con estacionalidad marcada por periodos más largos de secas y menos de lluvias hacen que sean más típico la vegetación de bosques secos, en este caso la cuenca del río Apurímac. De la misma forma a nivel de especie la presencia de Podocarpus glomeratus en nuestra área de estudio como la especie de mayor IVI, difiere notablemente con las otras áreas donde se reconocen como mayor IVI a especies propias de bosques montanos húmedos de la vertiente oriental de los andes.

Por tanto, la composición arbórea del SNA es en promedio relativamente de baja diversidad y riqueza en comparación a bosques de similar altitud como los bosques montanos de las vertientes orientales, pero son particulares por la composición de taxas que no se encuentran en otros ecosistemas, representados por los altos valores de IVI de Podocarpus glomeratus y especies de la familia Asteraceae.

La presencia de especies forestales claves- -en el SNA, hace resaltar a cinco que se encuentran en diferentes ámbitos de los andes tropicales de Sudamérica y están considerados en categorías de amenaza según el libro de la lista roja de los árboles de los bosques montanosTejedor et al. (2014), (tabla 11).

Tabla 11. Listado de especies de árboles del bosque montano - andino de Sudamérica, registrados en el presente estudio.

\begin{tabular}{|c|c|c|c|c|}
\hline Familia & Especie & $\begin{array}{c}\text { Categońa } \\
\text { UICN }\end{array}$ & $\begin{array}{c}\text { Lista roja } \\
\text { América latima }\end{array}$ & Distribudón \\
\hline Buxaceae & Stylocers laurifolium & $\mathrm{EN}$ & - & Bolivia, Eandor, P \\
\hline Escalloniacere & Escallonia resinsa & $\mathrm{vU}$ & NT & Bolivia, Perú \\
\hline Myrtaceae & Mrrianthes oreophila & $\mathrm{NT}$ & - & Bolivia, Perú \\
\hline Podocarpaceae & Podocappes glomratus & $\mathrm{NT}$ & $\mathrm{NT}$ & Eandor, Perú \\
\hline Verbenaceae & Duranta armata & - & NT & Bolivia, Pexú \\
\hline
\end{tabular}

\section{Biomasa}

El estudio de la biomasa y las reservas de carbono en comparación a los bosques Amazónicos, los bosques montanos en los andes están pobremente conocidos y estudiados (Kapelle \& Brown 2001; Gerardin et al. 2014b). Nuestros resultados a pesar que no muestran una diferencia significativa en la biomasa de cada parcela, corroborado también con las dos ecuaciones de Chave et al. 2005 y 2014, se observa que existen variaciones en cuanto a la estructura en cada sitio a pesar de estar relativamente cerca $(0.5-3 \mathrm{~km})$. Estas diferencias estadísticamente bajas, si son notables a nivel de estructura, donde los valores que resaltan son el número de individuos en la parcela PBA-4 que son casi tres veces que la parcela PBA-1 y casi el doble de PBA-3, con la dominancia de árboles de DAP menores que sin duda estas variaciones son por las condiciones quizá más favorables en altitudes más bajas, pero también está influenciada por el impacto antrópico. La biomasa en los bosques del SNA, están- 
-dominados por un grupo pequeño de taxas que aglomera los mayores volúmenes, de estas destaca Podocarpus glomeratus, el cual presenta el mismo patrón de biomasa en las cuatro parcelas, siendo extrema en algunas como sucede en la parcela PBA-3, donde P. glomeratus con un solo individuo de casi $4 \mathrm{~m}$ de Dap.

La variación de biomasa total de las 4 parcelas en el SNA de 135.0 hasta $141.5\left(\mathrm{t} \mathrm{ha}^{-1}\right)$, es corroborada por otros estudios donde la densidad de individuos con diámetros y alturas menores son dominantes en algunos sitios de mayor altitud como en la parte alta del Parque Nacional del Manu (Gerardin et al. 2014b; Moser et al. 2011). La cantidad de biomasa almacenada en los bosques andinos es variable, por ejemplo el SNA es similar a otros ubicados por encima de los 3000 msnm, como en Loja Ecuador, norte de Bolivia, sur de Perú y Venezuela con 53.9 hasta 197 (t ha ${ }^{-1}$ ), (Tabla 12), pero difiere notablemente de otras como en el sur de Ecuador a 2830 msnm se llegó a registrar más de 700 toneladas/hectárea (Graefe et al. 2008). En otras localidades se registraron cifras similares a los de bosques amazónicos como sucede en los bosques de "queña” Polylepis con $366\left(\mathrm{t} \mathrm{ha}^{-1}\right)$ y los bosques de aliso (Alnus acuminata) con 241 th/ha (Spracklen \& Righelato 2014). Por tanto, los bosques dominados por Podocarpus glomeratus "intimpa" del SNA muestran datos de biomasa que se pueden considerar como usuales (tabla 12).

En comparación a los bosques amazónicos como en el sur peruano la biomasa registrada para las cuatro parcelas del SNA es menor de aquellas ubicadas entre los 500 - $1500 \mathrm{msnm}$, donde se pueden hallar entre 250 a 300 toneladas/hectárea (Gerardin et al. 2014b).
Tabla 12. Comparación de datos de biomasa aérea entre localidades de bosques andinos.

\begin{tabular}{lccccl}
\hline \multicolumn{1}{c}{ Localidad } & $\begin{array}{c}\text { Altitud } \\
(\mathbf{m s n m})\end{array}$ & $\begin{array}{c}\text { Temperatura } \\
\left.\text { (prom. }{ }^{\circ} \mathbf{C}\right)\end{array}$ & $\begin{array}{c}\text { Precipitación } \\
\text { (to. anual mm) }\end{array}$ & $\begin{array}{c}\text { Biomasa } \\
\text { (ton/ha) }\end{array}$ & Refe rencia \\
\hline Ecuador (Loja) & 3060 & 8.6 & 4500 & 738 & Leuschner et al. (2007). \\
Ecuador (Loja) & 3060 & $\ldots$. & 4000 & 53.9 & Leuschner et al. (2007). \\
Ecuador (Oyacadhi) & 3200 & 10.5 & 2250 & 241 & Feshe et al. (2002) \\
Ecuador (Pichincha) & 3600 & 8 & 1500 & 366 & Feshe et al. (2002) \\
Venezuela (Mucuy) & $2640-3000$ & $\ldots$ & $\ldots$ & 197 & Delaney et al. (1997) \\
Sur de Perú & 3020 & 11.8 & 1776 & 94.08 & Girardin et al. (2013) \\
Sur de Perú & 3025 & 11.8 & 1570 & 129.3 & Girardin et al. (2013) \\
SNA (Perú)* & $3200-3390$ & 13 & 1200 & $144-150$ & Presente estudio \\
\hline
\end{tabular}

Estas variaciones pueden atribuirse a diversos factores abióticos como el suelo, temperatura y humedad, radiación solar (Benner et al. 2010; Bruijnzeel et al. 2010), aparentemente en el sur peruano incluido el SNA, el factor determinante es la humedad (Girardin et al. 2014b), ya que en los periodos de sequias y de lluvias estos regímenes son diferentes de otros lugares. Estos factores que influyen en general aún son poco conocidos localmente ya que aparentemente a nivel regional diversos estudios sugieren que la elevación no es la variable más importante de la variabilidad de biomasa, más bien las características físicoquímicas del suelo y la precipitación (Slik et al. 2010), los cuales están indirectamente relacionados a las variaciones en la gradiente altitudinal (Homeier et al. 2013, Báez et al. 2015; Girardin et al. 2010).

Finalmente podemos concluir que las biomasas de las cuatro parcelas en el Santuario Nacional del Ampay, muestra patrones similares a otros bosques montanos en el neotrópico, donde de acuerdo a la localidad pueden tener también variaciones extremas. Estos datos son corroborados en otros bosques lejanos como los bosques montanos del continente asiático como sugieren Spracklen \& Righelato (2014) en estudios recientes. En este contexto creemos que se deben de implementar y complementar otros estudios como de variables ambientales más locales, la habilitación de mayores áreas para la instalación parcelas permanentes y a la vez vincular estas- 
- con actividades de pobladores locales de las áreas de influencia, los cuales puedan ser fuente de generación de compensaciones económicas que eviten impactos aledaños a través de los mercados de Carbono.

\section{Implicancias para la conservación}

A pesar de que las áreas evaluadas de las parcelas en el SNA no muestran un impacto antrópico fuerte y aparente, este efecto contrariamente se hizo notorio al analizar el sotobosque que evidencio la presencia de suelo compactado, pocas poblaciones naturales de plántulas y de regeneración natural. La historia es similar con otros lugares donde el impacto antrópico en bosques, son evidenciados desde la época pre-incaica, continuándose en la época colonial hasta los tiempos actuales (Young 2009). En Abancay dentro del SNA hasta antes de su creación como Santuario Nacional, la tala del bosque era indiscriminada, la apertura de áreas de cultivo, plantaciones amplias de eucalipto y la actividad ganadera, por ello que en el sotobosque aún se observa caminos de ingreso de ganado y otras actividades actuales desarrolladas a los alrededores del SNA (PMSNA 2015). Los bosques montanos del SNA, forman parte de los bosques montanos del noroeste de los andes y las Yungas del sur peruano, los cuales están categorizados como amenazados dado que solo queda cerca del $50 \%$ como bosque primario o poco impactado (Tejedor et al. 2012).

A pesar de la actividad antrópica pasada, los parámetros poblacionales de estos bosques muestran que las especies nativas más representativas fueron: Podocarpus glomeratus, Myrcianthes oreophila, Hesperomeles gayana, y Myrsine andina, los cuales en otros ámbitos vienen siendo diezmados severamente, y las- -poblaciones del SNA son aparentemente mejor conservados en todo el ámbito del departamento de Apurímac que a su vez se encuentran en otras cuencas similares como el Urubamba entre las localidades de Ollantaytambo y el km 90 del ingreso al Santuario Histórico de Machupicchu, pero en menor grado de conservación (Tupayachi 2005), reconocidas como en peligro de desaparición por la alta demanda como leña y deforestación para el establecimiento de cultivos principalmente alrededores del valle sagrado de los Inkas (Tupayachi en prensa).

El presente estudio representa un punto de partida importante para el estudio y monitoreo de la dinámica de los bosques andinos en la región del sur peruano, principalmente de la región de Apurímac donde no se conoce otro similar. También se enfatiza la importancia que representa los bosques de intimpa ( $P$. glomeratus), corroborando al dato que no existe en otro lugar del Perú donde estas poblaciones estén mejor resguardadas. Por lo cual se recomienda llevar a cabo planes de manejo y conservación a largo plazo por parte de las autoridades municipales, del SNA vinculando directamente a las poblaciones locales que se encuentran en las áreas adyacentes al SNA.

\section{Agradecimientos}

Al Programa Bosques Andinos, implementado en consorcio entre HELVETAS-Swiss Intercooperation y el Consorcio para el Desarrollo Sostenible de la Ecorregión Andina (CONDESAN), a los fondos de la Agencia Suiza para el Desarrollo y la Cooperación (COSUDE), por habernos brindado los recursos y facilidades para llevar a cabo el presente estudio. A los técnicos y funcionarios del Santuario Nacional del Ampay, al Ing. Amilcar Osorio, Blgo. JaimeValenzuela y a- 
-todo el equipo de guardaparques por su apoyo logístico durante las evaluaciones en campo. A nuestro colega José Luis Marcelo Peña por la revisión del manuscrito. A los compañeros y colegas que nos apoyaron en la fase de campo y gabinete: Alex Ayte Turpo, Hans Candia, Naysha Huaman, Danae Arqque, Elias Quispe, Gladys Aymachoque y Giuliana Palomino de la UNSAAC, a Luis Carrasco, Ángela S. Peralta León y Susan Kelly C.G., de la Universidad tecnológica de los andes de Abancay por su apoyo en los trabajos de campo. A Yaneth Quispe por su apoyo con el recurso bibliográfico. A la Mgt. Fructuosa de la Torre que en su calidad de directora del herbario, hizo posible la cooperación entre el herbario CUZ y el proyecto, para el depósito de las muestras.

\section{Literatura citada}

Angiosperm Phylogeny Group 2016. "An update of the Angiosperm Phylogeny Group classification for the orders and families of flowering plants: APG IV". PDF. Botanical Journal of the Linnean Society , 181

(1): 1-20, $\underline{\text { doi: }}$ 10.1111/boj.12385, retrieved 201606-11.

Báez, S., Malizia, A., Carilla, J., Blundo, C., Aguiar, M. R., Aguirre, N., Aguirre, Z., Álvarez, E., Cuesta, F., Duque, A., Farfán Ríos, W., García-Cabrera, K., Grau, R., Homeier, J., Linares-Palomino, R., Malizia, L., Melo Cruz, O., Osinaga, O., Phillips, O. L., Reynel, C., Silman, M. R. \& Feeley, K. J. 2015. Large-scale patterns of turnover and basal area change in Andean forests. PLoS One, 2015. 10: p. e0126594.

Benner, J., Vitousek, P.M., Ostertag, R. 2010. Nutrient cycling and nutrient limitation in tropical montane cloud forests.

Bruijnzeel, L. A., Kappelle, M., Mulligan, M., \& Scatena, F. N. 2010. Tropical montane cloud forests: state of the knowledge and sustainability perspectives in a changing world, in: Tropical montane cloud forests: Science for Conservation and Management, edited by: Bruijnzeel, L.A.
-Scatena, F. N., and Hamilton, L. S., Cambridge University Press, 691-740.

Bush, M. B., B. C. S. Hansen, D. T. Rodbell, G. O. Seltzer, K. R. Young, B. Leo'n, M. B. Abbott, M. R. Silman \& Gosling, W. D. 2005. A 17,000year history of Andean climate and vegetation change from Laguna de Chochos, Peru. J. Quatern. Sci. 20: 703-714.

Caranqui, A. J. 2014. Diversidad y similitude arborea de los bosques montanos de la provincia de Chimborazo. XI Congreso de ciencia y Tecnología. Aespe. Ecuador. p. 11-17.

Chave, J., Andalo, C., Brown, S., Cairns, M., Chambers, J., Eamus, D., Folster, H., Fromard, F., Higuchi, N., Kira, T. et al. 2005. Tree allometry and improved estimation of carbon stocks and balance in tropicalforests. Oecologia 145:87-99.

Chave, J., Coomes, D. A., Jansens, S., Lewis, S. L., Swenson, N. G. y Zanne, A. E. 2009. Towards a worldwide wood economics spectrum. Ecology Letters 12:351-366.

Chave, J., Réjou-Méchain, M., Búrquez, A., Chidumayo, E., Colgan, M.S., Delitti, W.B.C. et al. 2014. Improved allometric models to estimate the aboveground biomass of tropical trees. Global Change Biology, 20, 3177-3190.

Condit, R., Foster, R.B., Hubbell, S.P. et al. 1998. Assessing forest di- versity on small plots: calibration using species-individual curves from 50 ha plots. Pages 247-268 in: Dallmeier, F. y J. A. Comiskey (eds.) Forest Biodiversity Research, Monitoring and Modeling. MAB Series Vol. 20, UNESCO, Paris, and Parthenon Publishing Group, N.Y.

Curtis, J.T. \& McIntosh, R.P. 1951. An Upland forest continuum in the prairie-Forest border region of Wisconsin. Ecology 32(3): 476-496.

Gentry, A. H. 1995. Patterns of diversity and floristic composition in neotropical montane forests. Pp 103-126 en H. Churchill S. P. B., E. Forero y J. L. Luteyn (Eds.). Neotropical Montane Forest 
-Bronx, N.Y., USA). Biodiversity and conservation of neotropical montane forests: Proceedings. New York Botanical Garden, Bronx, NewYork, USA.

Girardin, C.A.J., Farfan-Ríos, W., García K, Feeley, K.J., Jørgensen, P.M., AraujoMurakami , A., Pérez, L.C, Seidel, R., Paniagua, N., Fuentes Claros, A. et al. 2014b. Spatial patterns of above-ground structure, biomass and composition in a network of six Andean elevation transects. Plant Ecology and Diversity 7(1-2): 161-171.

Girardin, C.A.J., Aragão, LEOC., Malhi, Y., Huaraca, H. W., Metcalfe , D., Silvae, J.E. 2013. Fine root dynamics along an elevational gradient in tropical Amazonian and Andean forests. Global Biogeochemical Change 27:252-264.

Girardin, C. A. J., Malhi, Y., Aragão, L. E. O. C., Mamani, M., Huaraca, H, W., Durand, L., Feeleys, K. J., Rapp, J., Silva-Espejo, J. E., Silman, M., Salinas, N., \& Whittaker, R. J. 2010. Net primary productivity allocation and cycling of carbon along a tropical forest elevational transect in the Peruvian Andes, Glob. Change Biol., 16 , 3176-3192, doi:10.1111/j.13652486.2010.02235.x.

Graefe, S., Hertel, D. \& Leuschner, C. 2008. Fine root dynamics along a 2,000 $\mathrm{m}$ elevation transect in South Ecuadorian mountain rainforests. Plant and Soil 313:155-166.

Hammer, Ø., Harper, D.A.T., Ryan, P.D. 2001. PAST: Paleontological statistics software package for education and data analysis. http://palaeoelectronica.org/2001 1/past/issue 1 01.htm. Palaeontología Electrónica 4(1): 9pp.

Homeier, J., Hertel, D., Camenzind, T., Cumbicus, N. L., Maraun, M., Martinson, G. O., Poma, L. N., Rillig, M. C., Sandmann, D., Scheu, S., Veldkamp, E., Wilcke, W., Wullaert, H. \& Leuschner, C. 1013. Tropical Andean forests are highly susceptible to nutrient inputs - Rapid -an Ecuadorian Montane Forest, PLoSONE, 7, e47128, doi:10.1371/journal.pone.0047128.

IDMA/WWF. 1998. Diagnóstico del Plan Maestro del Santuario Nacional del Ampay. 102 p. In: Bruijnzeel LA, Scatena FN, Hamilton LS, editors. Tropical montane cloud forests. Cambridge (UK): Cambridge University Press. p. 90-100.

INRENA. 2006. Diagnóstico y Plan Maestro del Santuario Nacional de Ampay. Instituto 3Nacional de Recursos Naturales. Lima, Perú. Pp. 86.

Josse, C., Cuesta F., Navarro G., Barrena V., Cabrera E., Chacón-Moreno E., Ferreira W., Peralvo M., Saito J. \& Tovar A. 2009. Ecosistemas de los Andes del Norte y Centro. Bolivia, Colombia, Ecuador, Perú y Venezuela. Secretaría General de la Comunidad Andina, Programa Regional ECOBONA-Intercooperation, CONDESAN Proyecto Páramo Andino, Programa BioAndes, EcoCiencia, NatureServe, IAvH, LTAUNALM, ICAEULA, CDC-UNALM, RUMBOL SRL. Lima, Perú.

Kapelle, M., Brown, A.D. (eds). 2001. Bosques nublados del neotrópico. IUCN - INBio - Fundación ANA. Santo Domingo de Heredia (Costa Rica): Instituto Nacional de Biodiversidad.

Leuschner, C., Moser, G., Bertsch, C., Röderstein, M. \& Hertel, D. 2007. Large altitudinal increase in tree root/shoot ratio in tropical mountain forests of Ecuador. Basic and Applied Ecology 8:219-230.

La Torre-Cuadros, M.A. 2016. Estado del Arte Científico sobre el Manejo de Bosques Andinos en el Perú. Servicio Nacional Forestal y de Fauna Silvestre, Programa Bosques Andinos de la Agencia Suiza para el Desarrollo y la Cooperación - COSUDE, HELVETAS Swiss Intercooperation, CONDESAN. Pp. 96.

Magurran, A. E. 1988. Ecological diversity and its measurement.Princeton Univ. Press.

Moser, G., Leuschner, C., Hertel, D., Graefe, S., 
-the carbon budget of tropical mountain forests (S Ecuador): the role of the belowground compartment. Global Change Biology, 17, 2211-2226. doi: $10.1111 /$ j.1365-2486.2010.02367.x

Myers, N., Mittermeier, R.A., Mittermeier, C.G., da Fonseca, G.A. \& Kent, J. 2000. Biodiversity hotspots for conservation priorities. Nature 403:853-858.

Nina, Q. A. 2014. Composición, estructura y regeneración arbórea en dos parcelas permanentes del bosque nublado del Parque Nacional del Manu Paucartambo - Cusco. Tesis de grado - UNSAAC. Pp. 114.

Osinaga, O., Báez, S., Cuesta, F., Malizia, A., Carrilla, J., Aguirre, N. y Malizia, L. 2014. Monitoreo de diversidad vegetal y carbono en bosques andinos-Protocolo extendido. Protocolo 2 Versión 1. CONDESAN / IER-UNT / COSUDE. Quito, Ecuador.

Pennington, R.T., Lavin, M., Särkinen, T., Lewis, G.P., Klitgaard, B.B., Hughes, C.E. 2010. Contrasting plant diversification histories within the Andean biodiversity hotspot. Proceedings of the National Academy of Sciences 107:13783-13787.

Peralvo, M. \& F. Cuesta. 2015. Monitoreo de Dinámicas de Biodiversidad y Carbono: Estrategia de implementación de línea base - Santuario Nacional Ampay (SNA). Quito, CONDESAN, HELVETAS Y COSUDE.

Perea, M. J. 2005. Composición y estructura de especies arbóreas de un bosque montano, en el Parque Nacional Yanachaga Chemillen, Oxapampa Pasco - Perú. Tesis de grado. UNAP. Iquitos-Perú. Pp. 149.

Phillips, O.L. \& Miller, J. 2002. Alwyn H. Gentry Forest Transect Data Set. Miss. Bot. Garden. Monographs, Missouri.

Phillips, O., Baker, T., Feldpausch, T. \& Brienen, R. 2009. RAINFOR field manual for plot establishment and remeasurement. P. 1-24.

Plan maestro del Santuario Nacional del Ampay (PM-SNA). 2003. Aprobación del plan maestro-
-Santuario Nacional del Ampay. 2004-2008. Ministerio de Agricultura, Instituto Nacional de Agricultura, Intendencia de Áreas Naturales Protegidas, Apurímac, Perú. Pp. 126.

Plan Maestro Santuario Nacional del Ampay (PM-SNA). 2015. Aprobación del Plan Maestro Santuario Nacional del Ampay 2015-2019. Instituto Nacional de Agricultura, Intendencia de Áreas Naturales Protegidas, Apurímac, Perú. Pp. 55.

R Development Core Team. 2013. R Development Core Team. R: A Language and Environment for Statistical Computing.

Reynel, R.C. \& Antón, B.D. 2004. Diversidad y composición de la flora arbórea en un área de cumbre de colinas en bosque premontano: Fundo Génova-UNALM, valle de Chanchamayo 10001500 msnm. In: Antón, D. \& Reynel, C. (eds.). Relictos de bosque de excepcional diversidad en los Andes centrales de Perú. Perú Darwin Project-PBR APRODES-Universidad Nacional Agraria La Molina (UNALM), Lima. Pp. 143-186

Rivera, C. G. 2007. Composición florística y análisis de diversidad arbórea en un área de bosque montano en el Centro de Investigación Wayqecha, Kosñipata Cusco.Tesis de grado. UNALM- Lima. Pp. 121.

Slik, J.W. F., Aiba, S. I., Brearley, F. Q., Cannon, C. H., Forshed, O., Kitayama, K., Nagamasu, H., Nilus, R., Payne, J., Paoli, G., Poulsen, A. D., Raes, N., Sheil, D., Sidiyasa, K., Suzuki, E., and van Valkenburg, J. L. C. H. 2010. Environmental correlates of tree biomass, basal area, wood specific gravity and stem density gradients in Borneo's tropical forests, Global Ecol. Biogeogr., 19, 50-60.

Spracklen, D. V. \& Righelato, R. 2014. Tropical montane forests are a larger than expected global carbon store. Biogeosciences, 11(10), 2741-2754.

Tejedor, G. N., E. Álvarez Dávila, S., Arango Caro, Araujo-Murakami, A., Baldeón, A. et al. 2014. A Regional Red List of Montane Tree Species of the Tropical Andes: Trees at the top of the world. Richmond. Botanic Gardens Conservation- 
-International.Pp. 56.

Tejedor, N., Álvarez E, Arango S, Araujo A, Blundo C, Boza T, et al. 2012. Evaluación del estado de conservación de los bosques montanos en los Andes tropicales. Ecosistemas. 21(1-2):148-166.

Ter Steege, H., Pitman, N., Sabatier, D., Castellanos, H., van der Hout, P., Daly, D.C., Silveira, M., Phillips, O., Vásquez, R., van Ande, T., Duivenvoorden, J., Oliveira, A.A., Renske Ek, R., Lilwah, R., Thomas, R., van Essen, J., Baider, B., Maas, P., Mori, S., Terborgh, J., Nunez Vargas, P., Mogollón, H. \& Morawetz,W. 2003. A spatial model of tree adiversity and tree density for the Amazon. Biological Conservation 12:2255-2277.

Tupayachi, A. 2005. Flora de la Cordillera de Vilcanota. Arnaldoa 12: 126-144.

Webster, G.L. 1995. The Panorama of Neotropical Cloud Forests. Biodiversity and Conservation of Neotropical Montane Forests. P. 53-77.

Young, K. 2009. Andean land use and biodiversity: Humanized landscapes in a time of change. Annals of $t \quad h \quad e$ Missouri Botanical Garden. 96(3):492-507.

Young, K., Ulloa Ulloa, C., Luteyn, J. \& Knapp, S. 2002. Plant evolution and endemism in Andean South America: An introduction. The Botanical Review 68:4-21.

Young, K.R. \& León, B. 2007. Tree-line changes along the Andes: implications of spatial patterns and dynamics. Philosophical Transactions of the Royal Society B: Biological Sciences 362:263-272.

Young, K.R. 1998b. Composition and structure of a timberline forest in northcentral Perú. In: Dalmeier, F; Comiskey, J.A. (eds.). Forest Biodiversity in North, Central and South America and the Caribbean: Research and Monitoring. Man and the Biosphere Series Volume 22. Carnforth, Lancashire, Reino Unido, UNESCO and the Parthenon Publishing Group. p. 595-613.
Zanne, A.E., Lopez-Gonzalez ,G., Coomes, D.A., Ilic, J., Jansen, S., Lewis, S.L., Miller, R.B., Swenson, N.G., Wiemann, M.C. \& Chave, J. 2009. Data from: Towards a worldwide wood economics spectrum. Dryad Digital Repository. http://dx.doi.org/10.5061/dryad.234.

\section{Páginas web}

https://www.researchgate.net/publication/30288148

6 Outlook on climate change adaptation in th e Tropical Andes mountains\#pag:26:mrect: $(350.09110 .70,128.97,13.49)$

http://mobot.mobot.org

http://fieldmuseum.org http://www.theplantlist.org http://sinia.minam.gob.pe/normas/apruebancategorizacion-especies-amenazadas-flora-silvestre.

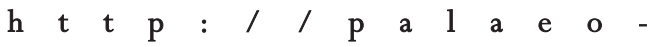
electronica.org/2001_1/past/issue1_01.htm 